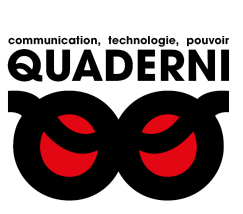

\title{
Quaderni
}

Communication, technologies, pouvoir

98 | Hiver 2018-2019

Humanités numériques : vers l'institutionnalisation

\section{Olivier Galland, Anne Muxel (dir.), La Tentation radicale. Enquête auprès des lycéens}

Julien Giry

\section{Q OpenEdition}

Édition électronique

URL : https://journals.openedition.org/quaderni/1495

DOI : 10.4000/quaderni.1495

ISSN : 2105-2956

Éditeur

Les éditions de la Maison des sciences de l'Homme

Édition imprimée

Date de publication : 5 février 2019

Pagination : 135-144

Référence électronique

Julien Giry, "Olivier Galland, Anne Muxel (dir.), La Tentation radicale. Enquête auprès des lycéens »,

Quaderni [En ligne], 98| Hiver 2018-2019, mis en ligne le 05 février 2019, consulté le 07 janvier 2022.

URL : http://journals.openedition.org/quaderni/1495; DOI : https://doi.org/10.4000/quaderni.1495 


\section{Compte rendu}

La Tentation radicale.

Enquête auprès des lycéens. Olivier Galland, Anne Muxel (dir.)

PUF, Paris, 2018

1. Oliver Galland, sociologue, est directeur de recherches au CNRS et rattaché au GEMASS. Anne Muxel, politiste et sociologue, est également directrice de recherches au CNRS rattachée au CEVIPOF/Sciences-Po. par Julien Giry

IDPSP

Université Rennes 1

Réalisée dans le cadre de «l'appel à propositions sur tous les sujets pouvant relever des questions posées à nos sociétés par les attentats et leurs conséquences » (p. 7) lancé par le CNRS à la suite des attaques terroristes de 2015, La tentation radicale prend la forme d'un ouvrage collectif de 455 pages dirigé par Olivier Galland et Anne Muxel ${ }^{1}$ et paru aux Presses Universitaires de France en avril 2018.

Sur le plan formel, après une brève introduction rédigée par ses deux codirecteurs, le livre propose aux lecteurs un « prologue » qui prend la forme d'un " carnet de terrain » où sont exposées les étapes successives de la recherche, les interrogations des chercheurs ainsi que les difficultés rencontrées. Ensuite, l'ouvrage se décompose en six chapitres qui envisagent tour à tour différents aspects relatifs au degré d'acceptation sociale de la radicalité religieuse et/ou politique parmi les lycéens. Enfin, une postface, une large bibliographie de près de 20 pages, et deux séries d'annexes détaillant les aspects méthodologiques et les outils de mesure utilisés complètent ce collectif.

Après avoir remercié les divers partenaires institutionnels ayant contribué au financement de la recherche, Galland et Muxel fixent dans l'introduction les enjeux essentiels de leur démarche. Ainsi, soulignent-ils leur ambition d'interroger, au travers de signes et manifestations divers et variés en intensité, les «degrés d'acceptation et de justification » de la radicalité religieuse et politique auprès des lycéens en allant au-delà des «travaux monographiques et qualitatifs $\gg($ p. 8). Dès le départ, deux choses sont claires : la prépondérance, sans être exclusive, des analyses quantitatives d'une part et ce n'est pas la 
radicalisation des lycéens qui sera ici étudiée mais bien leur acceptation de la radicalité idéelle et/ou actionnelle. D'emblée, les auteurs proposent alors de définir la radicalité comme « un ensemble d'attitudes ou d'actes marquant la volonté d'une rupture avec le système politique, économique, social et culturel, et plus largement avec les normes et les mours en vigueur dans la société. Elle atteint son point le plus extrême lorsqu'elle s'accompagne d'une justification de la violence » (p. 9). Afin d'étudier cette palette d'attitudes ou d'actes visant à légitimer la radicalité, les auteurs rappellent alors qu'ils ont dû opérer un certain nombre de choix méthodologiques forts, notamment dans la composition et le panachage de l'échantillon des enquêtés. Partant du principe que si la radicalité demeure un comportement minoritaire affectant toutes les religions mais que ses manifestations contemporaines sont les plus visibles au sein de l'islam, le panel a logiquement surreprésenté les lycéens de confession musulmane scolarisés dans des zones urbaines sensibles. Toutefois, afin d'assurer une diversité suffisamment représentative d'autres élèves de toutes origines et classes sociales et issus d'établissements ruraux et de centres-villes ont également été interrogés. Au total, un questionnaire a été administré à près de 7000 élèves de Seconde de toutes religions et classes sociales. Ceux-ci ont été interrogés dans une vingtaine d'établissements d'enseignement général, technologique et professionnel du Nord, d'Île-de-France, de Bourgogne et de PACA. Des entretiens individuels et collectifs (focus groups) sont par la suite venus compléter qualitativement les résultats de l'enquête. À titre de comparaison, les chercheurs ont également administré un questionnaire identique à un échantillon représentatif de 1800 jeunes âgés de 15 à 17 ans.

Le premier chapitre, « la radicalité en question », constitue le prolongement de l'introduction. Après avoir rappelé l'indétermination sémantique qui entoure la notion de radicalité, Galland et Muxel entreprennent un tour d'horizon des principales grilles de lectures et d'interprétation qui ont été avancées par les chercheurs, francophones et anglophones essentiellement, à propos de la radicalité politique puis religieuse. Sur le plan politique, un consensus semble se dessiner autour du caractère tout à la fois marginal, minoritaire et subversif de la radicalité, polymorphe dans ses formes et pratiques, mais dont l'ultima ratio réside dans la justification et le passage à la violence commise au nom de la cause. Toutefois, les auteurs insistent sur deux aspects : $1^{\circ}$ ) la nécessité de distinguer entre la radicalité exprimée sur le plan idéologique ou idéel et la radicalité actionnelle ou opérationnelle; $2^{\circ}$ ) le fait que si la 
2. Mohammed Hafez, Creighton Mullins, "The Radicalization Puzzle: A Theoretical Synthesis of Empirical Approaches to Homegrown Extremism", Studies in Conflict and Terrorism, $n^{\circ} 38,2015$, p. $958-975$. radicalité, l'extrémisme et la violence entretiennent des liens indéniables, ces trois termes ne sont pas interchangeables. Le premier déborde les deux autres. Sur le plan religieux, Galland et Muxel soulignent d'abord qu'il est impératif de ne pas confondre radicalité religieuse et fondamentalisme dans la mesure où la dimension violente n'est en rien consubstantielle à ce dernier. L'essentiel de cette partie consiste alors à discuter la pertinence des quatre critères facilitateurs ou prédictifs de radicalisation proposés par Hafez et Mullins ${ }^{2}$ : $1^{\circ}$ ) Les injustices subies ou ressenties par une frange de la population; $2^{\circ}$ ) Le rôle d'une idéologie englobante donnant un sens politique aux injustices subies ou ressenties et qui en désigne les coupables ; $3^{\circ}$ ) L'importance des réseaux de sociabilité entre des individus " ordinaires » et d'autres déjà « radicalisés »; $4^{\circ}$ ) La présence d'un environnement favorable. Dès lors, si comme le remarquent les auteurs, le lien entre l'exclusion et l'adhésion à une vision radicale de l'islam fait l'objet d'analyses discordantes, le critère d'adhésion à une idéologie hégémonique ou totalisante, en l'espèce le salafisme, est en revanche déterminant au même titre que le développement des réseaux socionumériques. $\mathrm{La}$ fin du chapitre prend alors acte du caractère polymorphe de la radicalité, de ses différences de degrés et de conséquences sociales. Au final, les auteurs insistent sur la nécessité de distinguer la radicalité protestataire, qui recouvre des formes non-conventionnelles de participation politique (manifestations, tags, votes pour les « extrêmes ", etc.), de la radicalité violente caractérisée par la justification de la violence comme répertoire d'action légitime. Au plan religieux, la radicalité procéderait de la rencontre entre une idéologie intransigeante et la légitimation de la violence.

Le deuxième chapitre, «Radicalité religieuse : de l'absolutisme à la violence », propose d'analyser les déterminants et les manifestions de cette radicalité religieuse. Aussi, s'il apparaît que les élèves musulmans, en particulier les garçons les moins intégrés au système scolaire, adhèrent davantage que les autres aux idées radicales et à la justification du recours à la violence pour des motifs religieux, reste à en expliquer les raisons. Deux facteurs semblent décisifs : l' « absolutisme » religieux et une plus grande tolérance à la violence et à la déviance ordinaires (non religieuse). Par absolutisme, le terme est repris par différents contributeurs de l'ouvrage, il convient d'entendre la combinaison $\mathrm{du}$ fondamentalisme religieux, y compris dans sa dimension orthopraxique, et du rejet de la sécularisation libérale, en particulier la croyance dans une supériorité architectonique du religieux. Cet absolutisme religieux apparaît au fil de l'enquête comme davantage développé chez les lycéens musulmans 
que parmi les autres groupes religieux testés et ceci presque indépendamment $\mathrm{du}$ facteur d'exclusion socioéconomique. La discrimination ressentie joue en effet un rôle certain mais néanmoins très secondaire. «L'effet spécifique de l'appartenance à l'islam dans l'engagement en faveur d'une idéologie absolutiste reste extrêmement puissant et il n'est que très peu affaibli par la prise en compte des hypothèses de victimisation ou de malaise identitaire » (p. 110). Ainsi, il ressort de l'enquête que si les lycéens de confession musulmane ont deux fois plus de chances de justifier l'emploi de la violence que les chrétiens, c'est parmi les musulmans absolutistes (30\%) que cette opinion est la plus rependue. Toutefois, au-delà de «l'effet islam », un second facteur joue un rôle déterminant dans la justification de la violence commise au nom de la religion : la tolérance à la violence et à la déviance ordinaires. En effet, souligne Galland, «le terreau de la radicalité religieuse n'est pas uniquement religieux [...] (il) est aussi manifestement associé à des prédispositions à la violence ou à la déviance détachées de motifs religieux » (p. 131). L'enquête fait alors apparaître que les lycéens les plus tolérants vis-à-vis de la violence et de la déviance ordinaires sont des garçons de confession musulmane qui sont peu intégrés au plan scolaire, qui déclarent subir des discriminations ethno-religieuses et qui expriment un malaise identitaire profond. En résumé, l'absolutisme religieux et une prédisposition à tolérer la violence sont les deux facteurs prédictifs essentiels de radicalité religieuse. Tous deux sont davantage développés parmi les élèves musulmans enquêtés.

Le troisième chapitre, « Les lycéens face aux attentats de 2015 », est un peu différent des deux premiers dans la mesure où il se présente comme une sorte d'étude de cas ou de monographie portant sur un aspect spécifique : la perception par les élèves des actes terroristes de janvier et novembre 2015 ainsi que des minutes de silence organisées en hommage aux victimes. Or, si les lycéens qui ne condamnent pas totalement les attentats et/ou qui ne se sentent pas réellement concernés par les minutes de silence ne se distinguent ni par leurs situations familiales ou socioéconomiques ni en raison d'un sentiment particulièrement aigu de discrimination ethno-religieuse, trois facteurs apparaissent en revanche déterminants : $1^{\circ}$ ) la tolérance à la violence et à la déviance ordinaires, $2^{\circ}$ ) être né à l'étranger, $3^{\circ}$ ) être de confession musulmane. Ainsi, si $24 \%$ des lycéens interrogés ne condamnent pas totalement les attentats de janvier 2015 menés contre les dessinateurs de Charlie Hebdo et $13 \%$ ceux de novembre au Bataclan notamment, ces chiffres grimpent respectivement à $58 \%$ et $34 \%$ chez les élèves les plus tolérants vis-à-vis de la 
3. Dans la mesure où il s'agit d'élèves de classe de Seconde n'ayant pas atteint l'âge légal du vote, il s'agit davantage de tester les potentialités au travers de questions telles que « Si vous aviez été en âge de voter à la prochaine élection présidentielle... ».

4. Jacques Ion, La fin des militants?, Paris, l'Atelier, 1997. violence ordinaire, à $41 \%$ et $21 \%$ chez ceux nés à l'étranger et, enfin, à $45 \%$ et $24 \%$ parmi les musulmans. Les propositions sont sensiblement les mêmes pour les minutes de silence. Toutefois, l'auteur du chapitre, Jean-François Mignot, appelle à trois nuances. D'abord, celui-ci remarque que les deux événements sont lus différemment par certains élèves. Bien que tous deux largement condamnés, les attentats de janvier font l'objet parmi les élèves d'une certaine forme de compréhension pour ne pas dire de mansuétude qui n'existe quasiment pas pour les attaques de novembre. Avec leurs caricatures du prophète, les dessinateurs du journal satirique l'auraient en quelque sorte « bien cherché » ou « provoqué » quand la foule du Bataclan ou des terrasses est perçue comme innocente et «tranquille». Ensuite, Mignot rappelle que le refus de condamner totalement les attentats n'est pas synonyme de leur approbation et encore moins de leur apologie. En effet, seulement 1 à $3 \%$ des enquêtés déclarent approuver les actes de terrorisme. Enfin, l'auteur souligne une distorsion statistique entre l'échantillon des lycéens enquêtés et celui représentatif de la population des 15-17 ans qui appelle à la prudence dans la mesure où seulement $5 \%$ de ce dernier ne condamnent pas totalement les attentats.

Le quatrième chapitre, «Radicalité politique : entre protestation et rupture », commence par souligner que "dans le domaine politique, la radicalité correspond à l'adhésion à des idées ou à des comportements supposant une opposition au jeu ordinaire de la politique et de ses institutions, ainsi qu'une stratégie frontale de contestation du système, cela selon une gradation allant de la protestation à la rupture » (p .205). En effet, Muxel distingue ici entre deux formes de radicalité, la protestation et la rupture, qui loin de se s'opposer s'inscrivent au contraire dans un continuum poreux où les frontières de l'une et de l'autre ne sont pas toujours bien lisibles. La radicalité de protestation, largement répandue parmi des lycéens malgré de fortes disparités de socialisation politique, renvoie ainsi à des comportements qui se situent à l'articulation entre participation politique conventionnelle, le vote essentiellement ${ }^{3}$, et non-conventionnelle : abstention, votes " extrêmes », manifestations, occupation de lycées, grèves, etc. Elle aspire à une transformation radicale de la société et du système politique. À titre d'exemple, si $28 \%$ des élèves interrogés déclarent s'intéresser à la politique, $42 \%$ des répondants n'auraient voté pour aucun des candidats présents à l'élection présidentielle de 2017. En effet, l'engagement politique des lycéens semble davantage « post-it » et marqué par des causes, la cause palestinienne, les luttes contre le racisme, la 
pauvreté, la faim dans le monde ou le terrorisme, que par un engagement électoral ou partisan. La radicalité de rupture, pour sa part, se caractérise par sa dimension subversive et sa propension à justifier le recours à la violence à des fins politiques. Celle-ci concerne une minorité de lycéens quand bien même 34\%, contre 15\% de l'échantillon représentatif des 15-17 ans, assurent qu'il est «acceptable dans certains cas de participer à une action violente pour défendre ses idées » (p. 245). Une fois ce constat dressé d'une socialisation et culture politiques radicales bien implantées au sein de la jeunesse lycéenne, l'auteure cherche à en dégager les facteurs explicatifs. Qu'il s'agisse de protestation ou de rupture, trois éléments communs semblent décisifs pour expliquer la radicalité lycéenne : un niveau d'intégration au système scolaire faible, un fort sentiment de discrimination et d'injustice et, enfin, une filiation politique penchant à gauche ou exprimant un sentiment de défiance face à la bipolarisation de la vie politique (« ni de droite, ni de gauche »). À l'inverse, les variables socioéconomiques jouent un rôle relativement faible dans l'adoption d'idées radicales. Cependant, au-delà de ces déterminants communs, il apparaît qu'un certain nombre de facteurs favorisent l'engagement vers l'une ou l'autre forme de radicalité. Ainsi, l'engagement protestataire est caractérisé un niveau élevé d'intégration au système politique marqué par une articulation entre formes de participation politique conventionnelles et non-conventionnelles ainsi qu'une adhésion aux valeurs du libéralisme politique et culturel. L'engagement de rupture, en revanche, est largement marqué par des dispositions favorables à la violence, à l'antilibéralisme et une adhésion à des valeurs et un leadership politique autoritaires. Il concerne davantage le genre masculin baigné dans une culture viriliste, des élèves scolarisés dans des filières professionnelles et très faiblement intégrés au système scolaire.

Dans le cinquième chapitre, "Frustration, discrimination et radicalité ", Laurent Lardeux propose d'interroger à deux niveaux les liens entre la frustration et la tolérance envers les idées et comportements radicaux : le décalage objectif entre les espoirs placés dans l'institution scolaire par les élèves malgré les inégalités et la manière dont, subjectivement, cette situation est traduite en termes d'injustices par les enquêtés. Ainsi, partant du constat que si tous les établissements considérés au cours de l'enquête sont marqués par une forte diversité ethnique et une forte disparité sociale, trois catégories de lycées se distinguent : ceux à forte mixité sociale, les établissements populaires et, enfin, les lycées très populaires. Toutefois, 
contrairement aux idées reçues, les élèves qui fréquentent les lycées « très populaires » présentent un degré d'intégration scolaire, c'est-à-dire la satisfaction exprimée et les aspirations placées dans l'École, à peu près identique à ceux scolarisés dans les autres types de lycées. En d'autres termes, «un établissement marqué par de très fortes inégalités sociales et fréquenté en majorité par des enfants d'immigrés (et d'ouvriers) ne comptera pas plus d'élèves faiblement intégrés au système scolaire qu'un lycée mixte socialement » (p. 283). Dans tous les cas, malgré des inégalités objectives entre les établissements, une large majorité des élèves place dans l'École un espoir d'ascension sociale et d'accession à un statut plus élevé que celui de ses parents. Au niveau subjectif, il apparaît que ces inégalités, si elles sont largement perçues individuellement et collectivement en termes d'injustices et de discriminations, la nature et la portée de cette perception varient fortement d'un groupe social à un autre. D'abord, les lycéens dont les deux parents sont français (métropole et outre-mer) ou originaires d'Europe du Sud se disent davantage discriminés à l'école en raison de leur apparence physique ou de leur manière de s'habiller. Ensuite, les élèves dont les deux parents sont originaires de pays non-européens mettent en avant des discriminations liées à leur religion, leur origine ou leur quartier. Ceci est particulièrement vrai pour les enfants issus de l'immigration turque puisque 30\% d'entre eux déclarent avoir déjà été personnellement victimes de telles discriminations. Enfin, le troisième groupe se compose des élèves issus de l'immigration maghrébine qui déclarent être victimes de discriminations liées à leurs religion et idées politiques. Une fois ce panorama dressé, Lardeux en vient alors à interroger les relations entre ces frustrations et l'adhésion aux idées et comportements radicaux. Sans surprise, l'enquête montre que les lycéens qui se sentent les plus discriminés en raison de leur origine, leur religion ou leur quartier sont ceux qui sont les plus enclins à justifier le recours à la violence quand bien même ce sentiment de révolte et d'injustice est davantage infra ou proto politique en ceci qu'il est largement dépourvu de perspectives réelles de changement social.

Si les auteurs de l'ultime chapitre, « Théories du complot et radicalité informationnelle ", soulignent de prime abord que seuls $9 \%$ des lycéens enquêtés se trouvent en situation de radicalité informationnelle comprise comme un sentiment de défiance vis-à-vis des médias traditionnels, l'adhésion à des théories conspirationnistes et la participation au flux informationnel de Daech, Vincent Cicchelli et Sylvie Octobre rapportent dans le même temps que près 
de $70 \%$ d'entre eux déclarent ne pas faire confiance aux médias alors même que la télévision, les chaînes d'information en continu en particulier, demeure leur source privilégiée d'information. Selon les auteurs, ce qui caractérise le régime d'information des jeunes est d'une part l'immédiateté symbolique et émotionnelle de l'image et une « apologie des idées personnelles comme critère de sélection » (p. 332) d'autre part. Ces éléments conduiraient les lycéens à adhérer plus facilement à des régimes alternatifs de vérités que constituent les théories du complot auxquelles, selon l'enquête, un jeune sur deux adhère au moins partiellement et $7 \%$ totalement. Six éléments ressortent de l'enquête : $1^{\circ}$ ) La mentalité conspirationniste est particulièrement développée chez les élèves issus de familles dominées économiquement ; $2^{\circ}$ ) Les élèves issus de minorités ethnoculturelles ayant subi personnellement des discriminations adhèrent plus que les autres aux théories du complot ${ }^{5}$; $3^{\circ}$ ) Les théories du complot sont davantage répandues parmi les élèves qui se sentent à la fois français et d'une autre origine (58\%), en particulier chez les lycéens musulmans $(64 \%) ; 4^{\circ}$ ) Les élèves qui trouvent la société la plus injuste adhèrent plus que les autres aux théories du complot; $5^{\circ}$ ) De même pour les élèves les plus insatisfaits de leur orientation; $6^{\circ}$ ) Les théories du complot se développent enfin sur fond de concurrence mémorielle et d'antisémitisme latent entre une histoire coloniale, la guerre d'Algérie notamment, qui serait largement ignorée et le massacre organisé des juifs européens dont la place serait largement exagérée. Les auteurs insistent alors particulièrement sur ce malaise identitaire, fait de discriminations ressenties, qui favorise, selon eux, la sur adhésion des lycéens musulmans aux théories du complot et les pousse, au même titre que les individus les plus tolérants à l'égard de la violence, à la participation au flux informationnel de Daech à deux niveaux : la simple consommation passive de vidéos et le relais de contenus (participation active). La combinaison entre l'effet religion et les prédispositions à légitimer la violence est alors décisive pour expliquer la radicalité informationnelle chez les lycéens.

Enfin, la « postface » signée de Galland et Muxel résume le principal enseignement de l'enquête. Bien qu'elle ne concerne qu'une minorité, la radicalité est néanmoins bien implantée au niveau idéel parmi les lycéens, notamment en ce qui concerne la justification du recours à la violence. La radicalité religieuse et l'acceptation de la violence commise au nom de la religion sont significativement plus développées chez les élèves de confession musulmane. Au niveau politique, si la radicalité touche un spectre d'élèves relativement

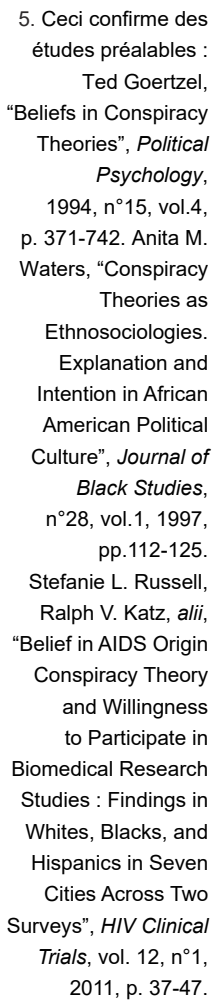


large, seule une infime minorité de garçons scolarisés dans les filières professionnelles et relégués socialement semblent concernée par une radicalité de rupture. En d'autres termes, pour les chercheurs, « la radicalité religieuse et la radicalité politique concernent a priori des segments de la jeunesse qui ne se recoupent pas forcément et obéissent à des facteurs différents » (p. 368). Pour conclure, les auteurs insistent sur trois aspects qui les ont particulièrement marqués au cours de l'enquête. D'abord, ils rappellent l'étonnant contraste entre le niveau élevé de défiance sociale et politique exprimé par les élèves et les espoirs qu'ils placent dans l'institution scolaire. Ensuite, ils insistent sur la surprise qu'a été pour eux la proportion non négligeable de lycéens exprimant un relatif sentiment d'indifférence émotionnelle face aux attentats survenus en 2015. Enfin, les auteurs ont été particulièrement marqués par l'ampleur et la place occupées par les théories du complot dans l'imaginaire social et politique des lycéens interrogés.

Si cet ouvrage collectif réalisé dans un contexte difficile se révèle de bonne facture, fort utile et fourmillant de données à la fois quantitatives et qualitatives inédites et de première importance brossant un état des lieux très complet de la tolérance des lycéens face aux idées et comportements radicaux, celui-ci appelle toutefois à quelques remarques.

Sur la forme, on regrettera que certains indicateurs, s'ils sont effectivement détaillés dans l'annexe 2 , ne soient pas présentés, même sommairement, lors de leur premier emploi dans l'ouvrage ou, à tout le moins, aient fait l'objet d'un renvoi vers l'annexe en question. Ceci est d'autant plus regrettable que les annexes sont relativement mal indexées et référencées dans la table des matières, ce qui les rend par conséquent plus difficiles à l'usage. Il en va ainsi, par exemple, de l'indicateur de «tolérance à la violence et à la déviance ordinaires » mobilisé dès le deuxième chapitre dont on aurait

6. On regrettera de manière plus secondaire que des établissements privés confessionnels n'aient pas été intégrés dans le champ de l'enquête et que les élèves juifs aient finalement été largement laissés de côté au prix d'une opposition binaire entre chrétiens et musulmans. aimé avoir un bref rappel dans le corps du texte ou dans une note de bas de page. De même, il faut attendre le cinquième chapitre pour que l'indicateur d'« intégration scolaire », pourtant utilisé dans les contributions préalables, soit précisément explicité.

Sur le fond, nous avons trois remarques essentielles ${ }^{6}$. D'abord, dans la mesure où il a été testé et démontré que la radicalité religieuse relève aussi d'éléments allogènes au fait religieux ou qui sont même de nature (proto) politique, on s'étonne alors que la radicalité politique envisagée au cours du quatrième 
chapitre n'ait pas été l'objet de croisements avec les variables religieuses ou que l'hypothèse d'une radicalité politique dont certaines prédispositions seraient corrélées avec « l'absolutisme religieux » n'ait pas été testée. Peutêtre est-ce pour éviter les controverses et polémiques que les résultats de telles corrélations, sans en préjuger, auraient immanquablement suscitées au sein de la communauté académique. Quoi qu'il en soit, ce manquement, à nos yeux essentiel, permet de questionner la conclusion du livre selon laquelle la radicalité religieuse et la radicalité politique concernent des segments différents de la jeunesse lycéenne. Ensuite, c'est finalement cette notion même d' « absolutisme religieux », construite à partir d'un croisement entre le fait de déclarer que seule sa propre religion est une vraie religion et la croyance en la supériorité de sa religion sur la science, qui peinent à convaincre. En effet, voir dans la première variable un signe de radicalité semble pour le moins excessif, surtout lorsqu'aucun équivalant n'est envisagé pour la radicalité politique. Il s'agirait de ne pas confondre laïcité et sécularisation ${ }^{7}$. Enfin, dans le sixième chapitre, il est dommageable que les enquêteurs n'aient testé qu'une seule théorie conspirationniste relative aux attentats du 11 septembre pour avancer que les élèves musulmans seraient plus sensibles que les autres au phénomène complotiste. En effet, plusieurs études ont montré que les acteurs sociaux ont davantage tendance à adhérer aux théories du complot et/ou à formuler des explications de type conspirationniste lorsque leur(s) groupe(s) d'appartenance(s) est (sont) concerné(s) ou incriminé(s) ${ }^{8}$. Or, nous le savons, les attentats du 11 Septembre ont été perpétrés par des acteurs musulmans. En conséquence, il aurait été intéressant pour consolider ou invalider l'affirmation selon laquelle les élèves musulmans sont plus sensibles que les autres aux théories du complot pour tester des théories conspirationnistes où des acteurs musulmans ne sont pas concernés, l'assassinat de JFK par exemple, ou des théories du complot incriminant, par exemple, des acteurs chrétiens afin de comparer les taux d'adhésion en fonction de l'appartenance confessionnelle.

Telles sont à nos yeux les principales remarques à formuler à l'endroit de cet ouvrage collectif, à la lecture fort agréable au demeurant, et qui n'enlèvent rien à ses qualités réelles. 